\title{
Structural Aspects of High-Efficiency Blue-Emitting 2,5-Bis(trimethylsilyl)thiophene-S,S-dioxide and Related Materials
}

\author{
Emilio Tedesco, Benson M. Kariuki, Kenneth D. M. Harris, ${ }^{1}$ and Roy L. Johnston \\ School of Chemistry, University of Birmingham, Edgbaston, Birmingham B15 2TT, United Kingdom \\ Olga Pudova \\ Latvian Institute of Organic Synthesis, Aizkraukles straße 21, Riga LV-1006, Latvia \\ Giovanna Barbarella \\ Consiglio Nazionale Ricerche, I.Co.C.E.A., Via Gobetti 101, 40129 Bologna, Italy \\ Elisabeth A. Marseglia \\ Cavendish Laboratory, University of Cambridge, Madingley Road, Cambridge CB3 OHE, United Kingdom \\ and \\ Giuseppe Gigli and Roberto Cingolani \\ Istituto Nazionale di Fisica della Materia, Dipartimento Ingegneria Innovazione, Università di Lecce, Via Arnesano, 73100 Lecce, Italy
}

Received April 10, 2001; in revised form June 21, 2001; accepted July 12, 2001

2,5-Bis(trimethylsilyl)thiophene-S,S-dioxide is a member of a wider family of materials of interest with regard to photoluminescence properties, and has been shown to exhibit a high efficiency of blue fluorescence emission in the solid state. The crystal structure of this material has been determined directly from powder X-ray diffraction data using the Genetic Algorithm technique for structure solution, followed by Rietveld refinement. The structural properties of this material are assessed in the context of a series of related derivatives of thiophene- $S, S$-dioxide. Potential energy calculations suggest that structural differences within this family of materials can be rationalized in terms of competing intermolecular interactions promoting different structure types. (C) 2001 Academic Press

\section{INTRODUCTION}

There is currently considerable interest in organic lightemitting devices, mostly due to the need to develop low-cost technology for display applications (1-3). In this field, the design and development of organic materials with high solid

\footnotetext{
${ }^{1}$ To whom correspondence should be addressed. E-mail: K.D.M. Harris@ bham.ac.uk.
}

state fluorescence efficiency is an important contemporary challenge (4). Although there has been significant progress in recent years (1-9), there is still considerable potential for developing new electroluminescent molecular materials and polymers, as well as for understanding and controlling the inter-relationships between their structural, electronic, and electro-optical properties in the solid state. In particular, it is now believed (10-13) that the geometric arrangement of these molecules in the solid state has a significant influence on their luminescence properties, and there is consequently increasing interest in understanding the intermolecular interactions and preferred modes of aggregation of these molecules in the solid state. In this regard, attention has focused on structural investigations of monomers and oligomers, which serve both as model compounds for the corresponding polymers and as potential materials for photophysical applications in their own right.

We report here the results of photophysical, structural, and computational investigations of 2,5-bis(trimethylsilyl)thiophene-S,S-dioxide (denoted 3; Scheme 1), which is a member of a broader family of compounds (denoted 1-5; Scheme 1). In this paper we report and discuss the crystal structure of 3, which has been determined directly from powder X-ray diffraction data using the Genetic Algorithm technique for structure solution. The structural properties of 


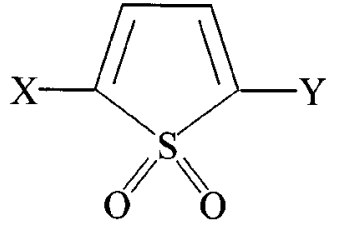

$1 \quad \mathrm{X}=\mathrm{Y}=\mathrm{C}\left(\mathrm{CH}_{3}\right)_{3}$

$2 \mathrm{X}=\mathrm{C}\left(\mathrm{CH}_{3}\right)_{3} \quad \mathrm{Y}=\mathrm{Si}\left(\mathrm{CH}_{3}\right)_{3}$

$3 \quad \mathrm{X}=\mathrm{Y}=\mathrm{Si}\left(\mathrm{CH}_{3}\right)_{3}$

$4 \mathrm{X}=\mathrm{Si}\left(\mathrm{CH}_{3}\right)_{3} \quad \mathrm{Y}=\mathrm{Ge}\left(\mathrm{CH}_{3}\right)_{3}$

$5 \quad \mathrm{X}=\mathrm{Y}=\mathrm{Ge}\left(\mathrm{CH}_{3}\right)_{3}$

SCHEME 1.

the other materials in this family have been determined independently $(14,15)$ from single-crystal X-ray diffraction data. Differences in the crystal structures and emission properties of 1-5 are discussed, and aspects of these structures are rationalized in terms of competing intermolecular interactions, which have been investigated by computational methods.

\section{EXPERIMENTAL}

Compound 3 was synthesized according to the procedure described in Ref. (16).

Photoluminescence measurements on powder samples of $\mathbf{3}$ and $\mathbf{5}$ were carried out using a $\mathrm{He}-\mathrm{Cd}$ laser operating at $325 \mathrm{~nm}$. Photoluminescence excitation measurements on 3 were carried out using a xenon lamp $(1000 \mathrm{~W})$ monochromated by a $0.5-\mathrm{m}$ single monochromator. The samples were mounted in a closed-cycle helium cryostat operating between $20 \mathrm{~K}$ and $300 \mathrm{~K}$, and the signal was detected by a 2-m double monochromator equipped with a cooled photon counter. Photoluminescence efficiencies $\left(\eta_{\mathrm{PL}}\right)$ were measured using an integrating sphere (17).

The synchrotron X-ray powder diffraction pattern of $\mathbf{3}$ was recorded at ambient temperature in transmission mode (capillary sample) on Station 2.3 at the Synchrotron Radiation Source (Daresbury Laboratory). The total range of $2 \theta$ was $3^{\circ}$ to $65^{\circ}(\lambda=1.3000 \AA)$ measured in steps of $0.01^{\circ}$ and collected over 6 hours. Structure solution was carried out using the Genetic Algorithm technique implemented in the program GAPSS (18-20). Rietveld refinement was carried out using the GSAS program (21).

Calculations of potential energies of crystals were carried out using the Cerius ${ }^{2}$ program (22) employing the CVFF95 potential energy parameterization (23-30). The Ewald summation method (31) was used, with a cut-off radius of $10 \AA$ for the repulsion term and cut-off radii of $15 \AA$ for the dispersion and electrostatic terms. In all calculations, the molecular geometry and the unit cell were allowed to relax under minimization of the total potential energy, although in all cases the bond lengths for a given molecule in different crystal structure types differed by less than $0.2 \%$, and the unit cell volumes differed by less than $5 \%$ from those in the experimental crystal structures.

\section{RESULTS AND DISCUSSION}

\section{Photophysical Measurements}

Compound 3 is characterized by high solid state fluorescence efficiency, emitting (as a microcrystalline powder) in the blue region $\left(\lambda_{\mathrm{PL}}=391 \mathrm{~nm}\right)$ with a fluorescence efficiency $\left(\eta_{\mathrm{PL}}\right)$ of $30 \%$. This fluorescence efficiency is substantially higher than those of oligothiophenes [which do not exceed a few percent in the solid state (32)] and polythiophenes [for which $\eta_{\mathrm{PL}}$ reaches $20 \%$ only in very few cases $\left.(33,34)\right]$. The emission lifetime of $\mathbf{3}$ is remarkably long (greater than $10 \mathrm{~ns})$. Figure 1 shows the photoluminescence spectrum and the photoluminescence excitation spectrum of $\mathbf{3}$. There is a bathochromic shift in the emission wavelength with respect to the absorption wavelength. On varying the temperature between $300 \mathrm{~K}$ and $30 \mathrm{~K}$, there is no observed change in the spectrum, suggesting that there are no significant structural changes in this temperature range.

The photoluminescence spectrum and fluorescence efficiency of the germanium derivative $\mathbf{5}$ in the solid state are very similar to those reported above for $\mathbf{3}$. As discussed below, $\mathbf{5}$ and $\mathbf{3}$ are isostructural, suggesting a strong correlation between structural and electro-optical properties. Preliminary measurements (35) on $\mathbf{1}, \mathbf{2}$, and $\mathbf{4}$ suggest that $\mathbf{1}$ and $\mathbf{4}$ have emission characteristics similar to those of $\mathbf{3}$ and 5 (comparable brilliance for $\mathbf{4}$; somewhat lower brilliance for 1). In contrast, 2 does not emit in the solid state. We note that oligomers of other thiophene- $S, S$-dioxides containing appropriate $\alpha$-functionalization and/or $\beta$-functionalization with alkyl or trialkylsilyl groups also exhibit high solid state fluorescence efficiency $(36,37)$.

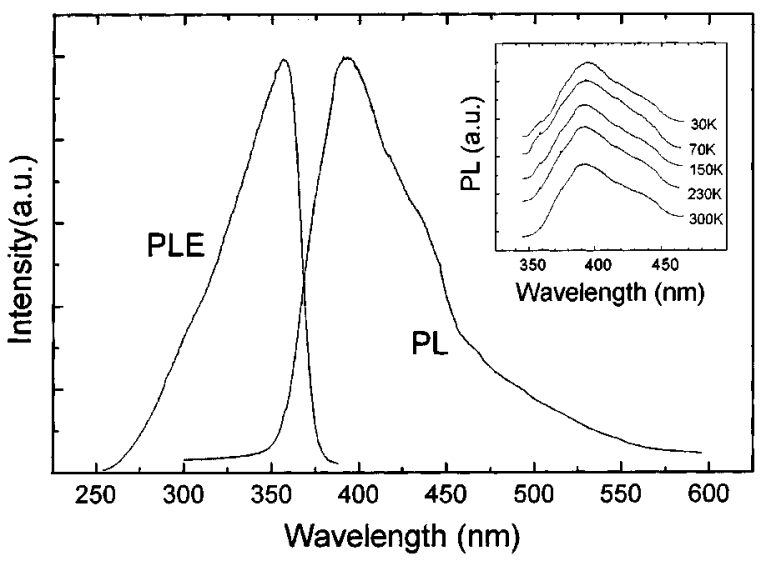

FIG. 1. Continuous wave photoluminescence (PL) and photoluminescence excitation (PLE) spectra of 3 . The inset shows the PL spectrum as a function of temperature. 


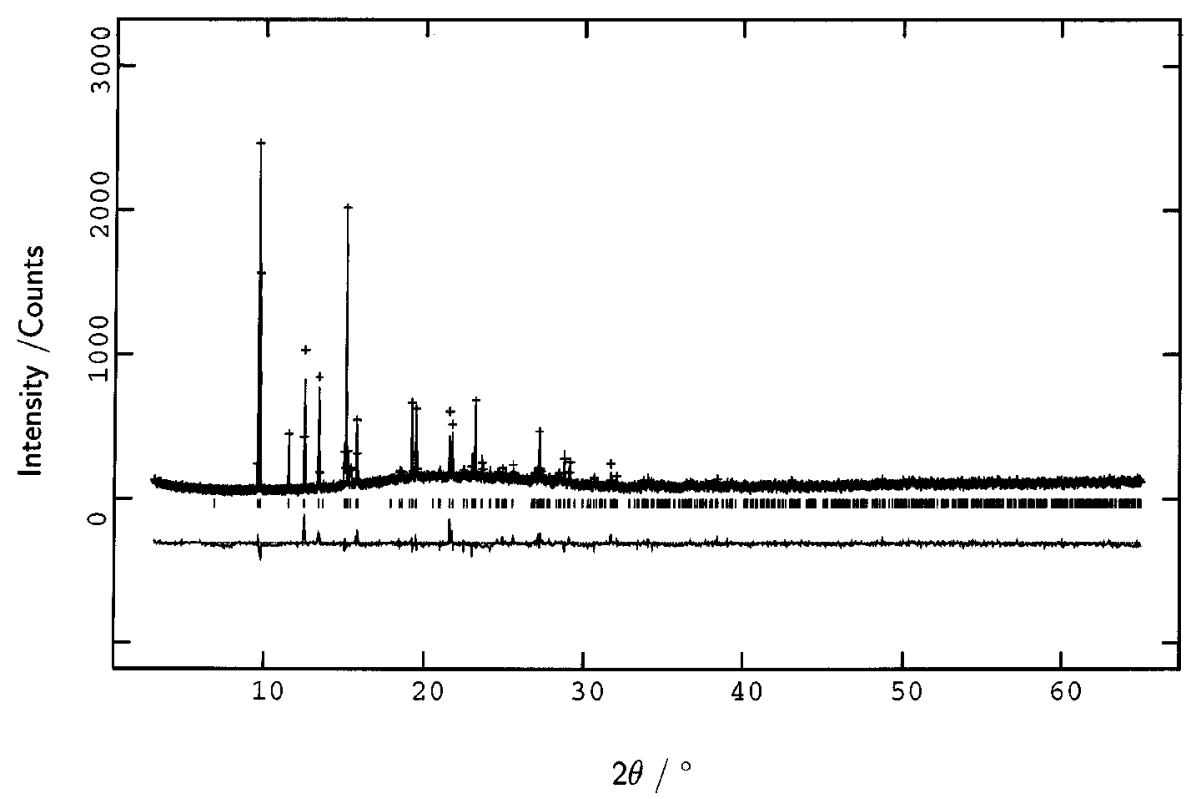

FIG. 2. Experimental ( + marks), calculated (solid line), and difference (lower line) powder X-ray diffraction profiles for the final Rietveld refinement of 3 .

\section{Structure Determination}

To explore relationships between the electro-optical properties and structural properties in the solid state, the crystal structure of $\mathbf{3}$ has been determined. As single crystals of appropriate size and quality for conventional singlecrystal X-ray diffraction studies could not be prepared, we have instead exploited recent developments in the techniques available for solving crystal structures directly from powder diffraction data (38-41).

The synchrotron X-ray powder diffraction pattern of 3 was indexed (using data up to $2 \theta=25^{\circ}$ ) by the program TREOR (42) giving the unit cell: $a=6.49 \AA, b=15.58 \AA$, $c=15.37 \AA, \alpha=\beta=\gamma=90^{\circ}$. Systematic absences were consistent with the orthorhombic space groups $P n 2_{1} m$ and Pnmm, and density considerations suggested that there are four molecules in the unit cell.

The crystal structure was solved from the powder diffraction data using the Genetic Algorithm (GA) method, details of which are described elsewhere $(18-20,43,44)$. The structure solution calculation was carried out in space group $P n 2_{1} m$, and involved the evolution of 200 generations of a population of 100 structures. In each generation, 100 offspring (50 pairs of parents) and 20 mutants were generated. The structural fragment was constructed using standard bond lengths and bond angles and comprised two half-molecules in the asymmetric unit, positioned appropriately with respect to the mirror plane. Rotation about the $\mathrm{C}$ (ring) $-\mathrm{SiMe}_{3}$ bonds represented a total of two intramolecular degrees of freedom.
The best structure solution (i.e., the structure with lowest $R_{\text {wp }}$ in the final generation in the GA calculation) was taken as the starting structural model for Rietveld refinement. All atoms were refined, with standard geometric restraints applied to bond lengths and bond angles. Hydrogen atoms were introduced into the structural model in calculated positions. A common isotropic displacement parameter was refined for all nonhydrogen atoms, and a preferred orientation parameter was refined in the final stages. After carrying out the refinement in space group $P n 2_{1} m$, it was recognized that space group Pnmm provides a better description of the structure, and subsequent refinement calculations were carried out in this space group. Indeed, an energy minimization calculation on the structure solution obtained in space group $P n 2{ }_{1} m$ suggests that an additional mirror plane (corresponding to space group Pnmm) is present. In space group Pnmm, the molecule is located at the intersection of two perpendicular mirror planes (the two-fold axis of the thiophene ring system runs along the intersection of these mirror planes). The final Rietveld refinement (Fig. 2) gave $R_{\mathrm{wp}}=10.85 \%$ and $R_{\mathrm{p}}=8.09 \%$ (6200 profile points; 617 reflections; 40 variables). The final refined structure is shown in Fig. 3, and fractional coordinates are given in Table 1.

In the crystal structure of 3 (Fig. 3), the molecules are arranged in an edge-to-face manner. The structure contains a parallel alignment of the molecular dipoles along the $b$-axis, whereas in the direction of the $c$-axis, adjacent molecules are arranged in an antiparallel manner. These structural features may be interpreted in terms of optimization of intermolecular electrostatic interactions. 


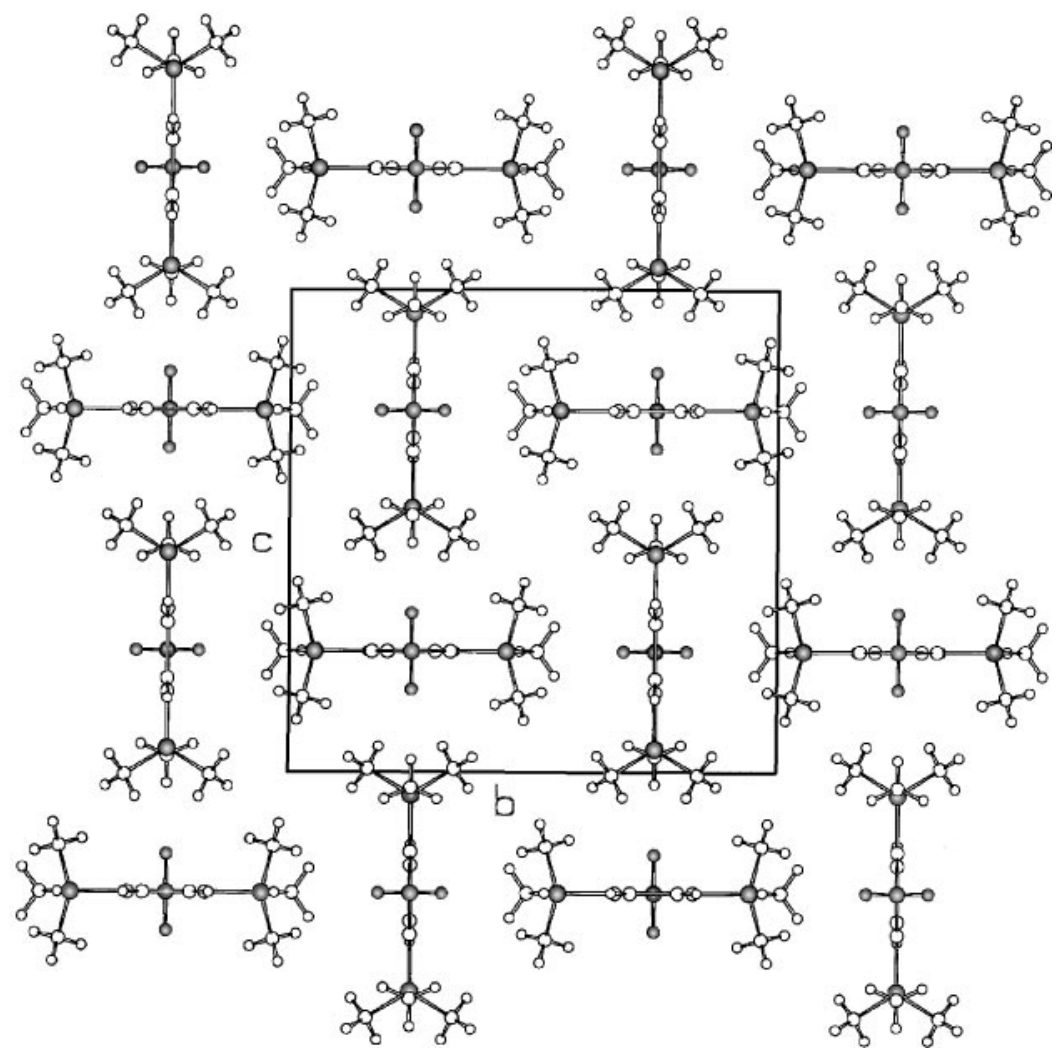

FIG. 3. The crystal structure of $\mathbf{3}$ viewed along the $a$-axis (sulfur, oxygen, and silicon atoms are shaded).

The crystal structures of $4\left(\mathrm{SiMe}_{3}, \mathrm{GeMe}_{3}\right)$ and $5\left(\mathrm{GeMe}_{3}\right.$, $\mathrm{GeMe}_{3}$ ), which were reported (15) subsequent to our completion of the structure determination of $\mathbf{3}$ reported here, are found to be isostructural with that of $3\left(\mathrm{SiMe}_{3}, \mathrm{SiMe}_{3}\right)$. Table 2 summarizes relevant crystal data for $\mathbf{3}$ and for the crystal structures of $\mathbf{1}, \mathbf{2}, \mathbf{4}$, and $\mathbf{5}$.

TABLE 1

Fractional Coordinates of the Non-Hydrogen Atoms in the Final Refined Crystal Structure of 3

\begin{tabular}{lclcc}
\hline Atom & $x$ & \multicolumn{1}{c}{$y$} & $z$ & $U_{\text {iso }} / \AA^{2}$ \\
\hline $\mathrm{O}(1)$ & $0.329(2)$ & $0.8165(5)$ & $\frac{1}{4}$ & $0.079(3)$ \\
$\mathrm{S}(2)$ & $\frac{1}{4}$ & $0.9380(2)$ & $\frac{1}{4}$ & $0.079(3)$ \\
$\mathrm{C}(3)$ & $\frac{1}{4}$ & $1.1166(1)$ & $0.17012(5)$ & $0.079(3)$ \\
$\mathrm{C}(4)$ & $\frac{1}{4}$ & $1.3185(1)$ & $0.2015(7)$ & $0.079(3)$ \\
$\mathrm{Si}(5)$ & $\frac{1}{4}$ & $1.04708(8)$ & $0.05402(4)$ & $0.079(3)$ \\
$\mathrm{C}(6)$ & $\frac{1}{4}$ & $1.2785(1)$ & $-0.00972(5)$ & $0.079(3)$ \\
$\mathrm{C}(7)$ & $0.34565(6)$ & $0.8982(1)$ & $0.02966(5)$ & $0.079(3)$ \\
$\mathrm{O}(8)$ & $\frac{1}{4}$ & $0.9028(5)$ & $0.8144(3)$ & $0.079(3)$ \\
$\mathrm{S}(9)$ & $\frac{1}{4}$ & $0.7536(2)$ & $\frac{3}{4}$ & $0.079(3)$ \\
$\mathrm{C}(10)$ & $0.33537(5)$ & $0.5858(1)$ & $\frac{3}{4}$ & $0.079(3)$ \\
$\mathrm{C}(11)$ & $0.30756(7)$ & $0.3798(1)$ & $\frac{3}{4}$ & $0.079(3)$ \\
$\mathrm{Si}(12)$ & $0.45250(4)$ & $0.66390(1)$ & $\frac{3}{4}$ & $0.079(3)$ \\
$\mathrm{C}(13)$ & $0.50696(5)$ & $0.5357(1)$ & $0.83756(6)$ & $0.079(3)$ \\
$\mathrm{C}(14)$ & $0.46795(6)$ & $0.9260(1)$ & $\frac{3}{4}$ & $0.079(3)$ \\
\hline
\end{tabular}

We now compare the crystal structure of 3 with the known crystal structures of $\mathbf{1}\left(\mathrm{CMe}_{3}, \mathrm{CMe}_{3}\right)$ and $\mathbf{2}\left(\mathrm{CMe}_{3}\right.$, $\mathrm{SiMe}_{3}$ ). In the structure of 1 (Fig. 4a), the molecules are arranged in an edge-to-face manner, but with a slight tilt of the two-fold axis of the thiophene ring relative to that in the structure of 3 . In contrast, the structure of 2 (Fig. $4 \mathrm{~b}$ ) is substantially different from those of $\mathbf{1}$ and $\mathbf{3}$, with a different overall arrangement of the thiophene rings. Furthermore,

TABLE 2

Summary of Crystal Data for Compounds $1-5^{a}$

\begin{tabular}{lccccc}
\hline & $\mathbf{1}$ & $\mathbf{2}$ & $\mathbf{3}$ & $\mathbf{4}$ & $\mathbf{5}$ \\
\hline$a(\AA)$ & 10.210 & 11.257 & $6.4917(3)$ & 6.513 & 6.525 \\
$b(\AA)$ & 9.480 & 6.439 & $15.5778(3)$ & 15.406 & 15.505 \\
$c(\AA)$ & 6.400 & 19.735 & $15.3702(3)$ & 15.556 & 15.600 \\
$\beta\left({ }^{\circ}\right)$ & 90 & 92.68 & 90 & 90 & 90 \\
$V_{\text {cell }}\left(\AA^{3}\right)$ & 619.5 & 1428.9 & 1554.4 & 1560.8 & 1578.3 \\
$Z$ & 2 & 4 & 4 & 4 & 4 \\
Space group & $P 2{ }_{1} 2$ & $C 2 / c$ & Pnmm & Pnmm & Pnmm \\
$V_{\text {mol }}\left(\AA^{3}\right)$ & 207.7 & 234.2 & 235.6 & 240.2 & 245.4 \\
PC & 0.67 & 0.66 & 0.61 & 0.62 & 0.62 \\
Reference & 14 & 15 & This paper & 15 & 15 \\
\hline
\end{tabular}

${ }^{a} \mathrm{PC}$ denotes the packing coefficient, which is defined as $Z V_{\mathrm{mol}} / V_{\text {cell }}$. In this expression, $V_{\mathrm{mol}}$ is the molecular volume, $V_{\text {cell }}$ is the unit cell volume, and $Z$ is the number of molecules in the unit cell. 


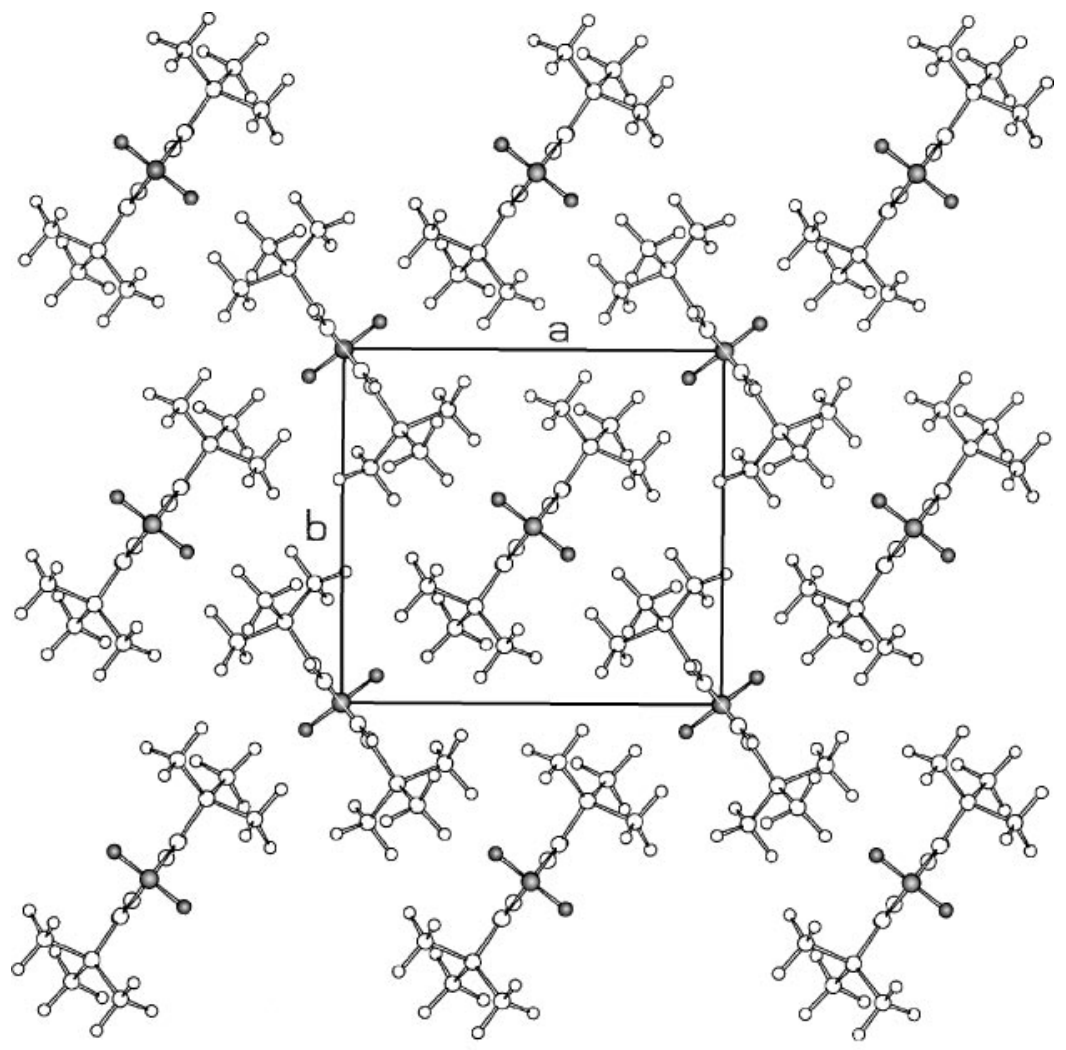

(a)

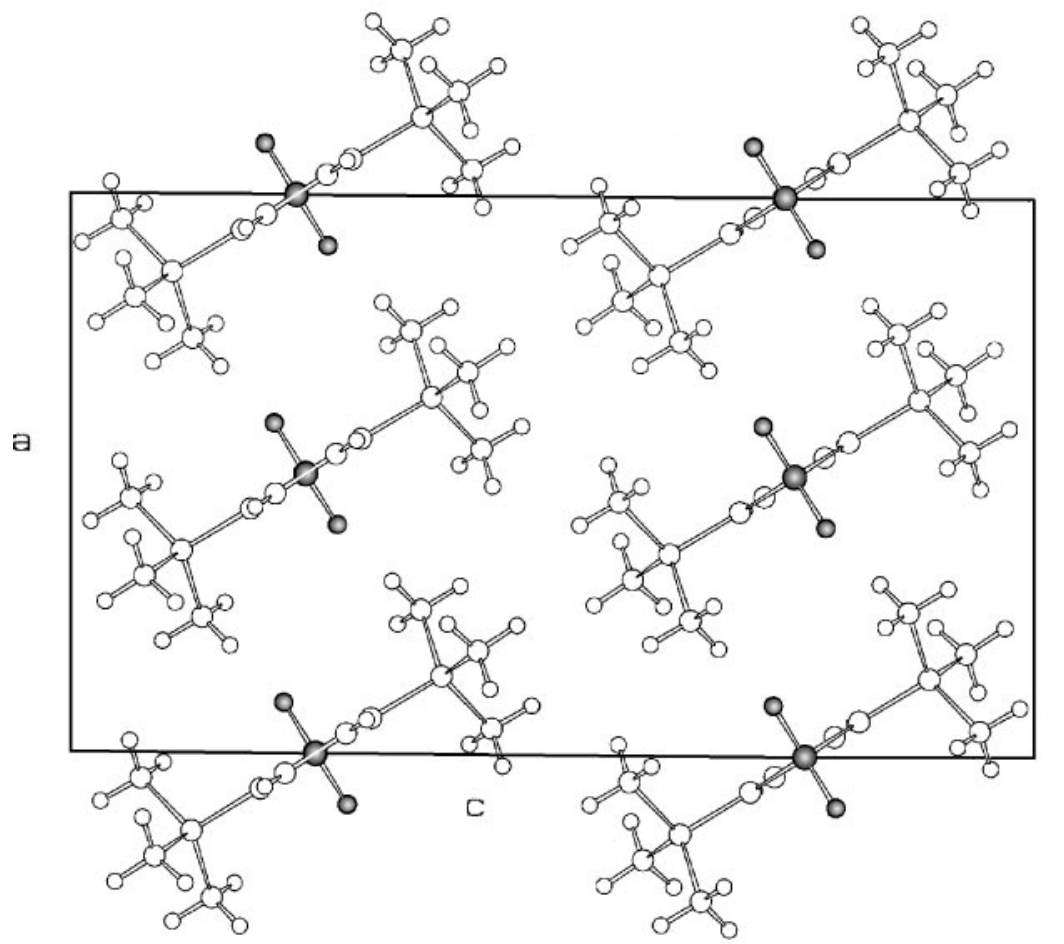

(b)

FIG. 4. (a) The crystal structure of $\mathbf{1}$ viewed along the $c$-axis (sulfur and oxygen atoms are shaded). (b) The crystal structure of 2 viewed along the $b$-axis (sulfur and oxygen atoms are shaded). 
the structure of $\mathbf{2}$ is disordered, and each molecule in the average crystal structure has one $\mathrm{CMe}_{3}$ side group with half occupancy and one $\mathrm{SiMe}_{3}$ side group with half occupancy on each side of the molecule. The $\mathrm{C}$ and $\mathrm{Si}$ atoms of these side groups are located at the same average position, and the $\mathrm{C}$ (ring) $\mathrm{C} / \mathrm{Si}$ distances therefore represent an average of $\mathrm{C}-\mathrm{C}$ and $\mathrm{C}-\mathrm{Si}$ bond lengths. Chains of molecules run along the direction of the $a$-axis, with neighboring molecules interacting through $\pi \cdots \pi$ stacking. Further interactions along the $c$-axis may be interpreted in terms of interlocking of methyl groups. In such disordered average crystal structures, we cannot determine a priori whether the structure comprises (a) ordered domains containing only one molecular orientation and an equal amount of ordered domains containing only the other molecular orientation, or (b) a situation in which, in each region of the crystal, each individual molecule has a $50 \%$ probability of being in one or other of the two orientations. In case (a), the disorder in the average structure arises from averaging over the different domains, whereas in case (b), the disorder exists at the molecular level. The packing coefficients (Table 2) decrease from $1(0.67)$ to $3(0.61)$, with very similar values of the packing coefficients for $\mathbf{3}, \mathbf{4}$, and 5 .

The similarity between the crystal structures of $\mathbf{3}, \mathbf{4}$, and 5 is reflected in their emission properties. For example, as discussed above, $\mathbf{3}$ and $\mathbf{5}$ have identical fluorescence spectra and fluorescence efficiencies, whereas no emission is observed for $\mathbf{2}$ in the solid state.

\section{Potential Energy Calculations}

In this section, we apply computational approaches to obtain more insight into the observed structural properties of compounds 1-5 in the solid state. Since the crystal structures of $\mathbf{3}, \mathbf{4}$, and 5 are essentially identical, the family of compounds 1-5 exhibit only three different structure types, exemplified by the experimental crystal structures of compounds $\mathbf{1}, \mathbf{2}$, and $\mathbf{3}$.

The potential energy calculations described below estimate the total potential energy $\left(E_{\text {tot }}\right)$ of a crystal in terms of intramolecular and intermolecular contributions. The intramolecular contribution $\left(E_{\text {intra }}\right)$ comprises bond stretching terms, bond bending terms, and cross-terms, whereas the intermolecular contribution comprises van der Waals $\left(E_{\mathrm{vdW}}\right)$ and electrostatic $\left(E_{\text {elec }}\right)$ terms. In order to compare the energetic properties of the three different structure types, we consider calculations for a given type of molecule in each of the three different structure types. As the calculations require ordered periodic structures, such calculations cannot be carried out for the observed (average) crystal structure of $\mathbf{2}$, and the unsymmetric molecule $\mathbf{2}$ cannot be incorporated into the crystal structures of $\mathbf{1}$ and $\mathbf{3}$. Nevertheless, the symmetric molecules $\mathbf{1}$ and $\mathbf{3}$ can be inserted into the crystal structure type observed for 2 . Thus, we have carried out calculations for molecule 1 (i.e., with two $\mathrm{CMe}_{3}$ side groups) in each of the three structure types for compounds $\mathbf{1}, \mathbf{2}$, and $\mathbf{3}$ (these calculations are denoted $\{\mathbf{1}\}_{\mathbf{1}},\{\mathbf{1}\}_{\mathbf{2}}$, and $\{\mathbf{1}\}_{3}$, respectively), and for molecule $\mathbf{3}$ (i.e., with two $\mathrm{SiMe}_{3}$ side groups) in each of the three structure types for compounds 1, 2, and $\mathbf{3}$ (these calculations are denoted $\{\mathbf{3}\}_{1}$, $\{\mathbf{3}\}_{2}$, and $\{\mathbf{3}\}_{3}$, respectively). The experimental crystal structures of $\mathbf{1}$ and $\mathbf{3}$ correspond to $\{\mathbf{1}\}_{1}$ and $\{\mathbf{3}\}_{3}$, respectively. The results of the calculations are summarized in Table 3.

In comparative terms, the results obtained for molecule 1 in each of the three crystal structure types \{\}$_{1},\{\}_{2}$, and \{\}$_{3}$ mirror the results obtained for molecule 3 in each of these crystal structure types. Thus, for both molecules $\mathbf{1}$ and 3, the following general observations are made: (i) structure type \{\}$_{1}$ has the lowest value of $E_{\text {tot }}$, with the following order of increasing $E_{\mathrm{tot}},\{\}_{1}<\{\}_{3}<\{\}_{2}$; (ii) this ordering of the values of $E_{\text {tot }}$ is dominated by the electrostatic contribution $E_{\text {elec }}$, which is the energy contribution that exhibits the largest variation in magnitude across the three different structure types, and follows the same trend as the values of $E_{\text {tot }}$-thus, in order of increasing $E_{\text {elec }},\{\}_{1}<\{\}_{3}<\{\}_{2}$; (iii) structure type \{\}$_{2}$ has the lowest value of $E_{\mathrm{vdW}}$, with the following order of increasing $E_{\mathrm{vdW}},\{\}_{2}<\{\}_{1}<\{\}_{3}$; (iv) structure type \{\}$_{2}$ has the highest density, with the following order of decreasing density, \{\}$_{2}>\{\}_{1}>\{\}_{3} ;(v)$ in terms of the variation, across the three structure types, of

TABLE 3

Total Potential Energy $\left(E_{\text {tot }}\right)$ for Molecules 1 and 3 in Each of the Structure Types Found Experimentally for Compounds 1,2 , and $3^{a}$

\begin{tabular}{|c|c|c|c|c|c|c|}
\hline Structure & $E_{\mathrm{tot}}\left(\mathrm{kcal} \mathrm{mol}^{-1}\right)$ & $E_{\text {intra }}\left(\mathrm{kcal} \mathrm{mol}^{-1}\right)$ & $E_{\mathrm{vdW}}\left(\mathrm{kcal} \mathrm{mol}^{-1}\right)$ & $E_{\text {elec }}\left(\mathrm{kcal} \mathrm{mol}^{-1}\right)$ & $\rho\left(\mathrm{g} \mathrm{cm}^{-3}\right)$ & $V_{\text {cel1 }} / Z\left(\AA^{3}\right)$ \\
\hline$\{\mathbf{1}\}_{1}$ & -266.0 & 133.0 & -63.0 & -336.0 & 1.171 & 323.8 \\
\hline$\{1\}_{2}$ & -262.4 & 132.9 & -64.7 & -330.6 & 1.194 & 317.6 \\
\hline$\{\mathbf{1}\}_{3}$ & -264.9 & 129.4 & -60.2 & -334.1 & 1.148 & 330.3 \\
\hline$\{3\}_{1}$ & -444.3 & 131.3 & -121.2 & -454.4 & 1.196 & 361.7 \\
\hline$\{3\}_{2}$ & -437.8 & 131.7 & -122.9 & -446.6 & 1.224 & 353.5 \\
\hline$\{3\}_{3}$ & -440.9 & 129.5 & -117.4 & -453.0 & 1.163 & 372.1 \\
\hline
\end{tabular}

\footnotetext{
${ }^{a}$ The individual energy contributions $E_{\mathrm{intra}}, E_{\mathrm{vdW}}$, and $E_{\text {elec }}$ are also given, together with the density $(\rho)$ and the volume per molecule $\left(V_{\text {cell }} / Z\right)$ in the unit
} cell. 
the magnitudes of the three contributions to the total energy, the variation is smallest for $E_{\text {intra }}$ and largest for $E_{\text {elec }}$.

For molecule 1, the experimentally observed crystal structure $\{\mathbf{1}\}_{1}$ has the lowest value of $E_{\text {tot }}$, whereas for molecule $\mathbf{3}$, the structure with the lowest value of $E_{\text {tot }}$ is $\{\mathbf{3}\}_{1}$, rather than the experimentally observed crystal structure $\{\boldsymbol{3}\}_{3}$ (which is the structure type giving the second lowest value of $E_{\text {tot }}$ for molecule 3). Thus, while the experimentally observed structure for molecule $\mathbf{1}$ is predicted from our calculations to be the polymorph of lowest energy (with respect to the particular potential energy parameterization used in our calculations) among the three structure types investigated, the experimentally observed structure for molecule $\mathbf{3}$ is predicted from these calculations to be a metastable polymorph. It is clear from the results of these calculations that optimization of the intermolecular electrostatic interaction is the dominant factor that discriminates structure type \{\}$_{1}$ as the preferred structure for both molecules $\mathbf{1}$ and 3. On the other hand, structure type \{\}$_{2}$ has the most favorable van der Waals interactions, both for molecules $\mathbf{1}$ and $\mathbf{3}$, and is the structure type of highest density in each case.

The fact that the experimentally observed crystal structure for compound $\mathbf{2}$ is a substantially different structure from those of the other members of the family may be related intrinsically to the unsymmetric nature of this molecule and/or the disorder in this structure, and thus the calculations presented here (which have considered symmetric molecules in ordered crystal structures) cannot shed light directly on this issue. Nevertheless, the fact that compound 2 forms the structure type for which the van der Waals contribution is apparently most favorable may be related in some manner to the important steric requirement (encountered only for 2) of accommodating side groups of different size within the crystal.

\section{CONCLUDING REMARKS}

The crystal structure of 2,5-bis(trimethylsilyl)thiophene$S, S$-dioxide (3) has been determined through the use of modern techniques for solving structures directly from powder X-ray diffraction data, and the structural properties have been rationalized in comparison with those of related derivatives of thiophene-S,S-dioxide. Compounds $\mathbf{3}, \mathbf{4}$, and 5 exhibit very similar emission features in the solid state, and the fact that the crystal structures are isostructural is in support of a strong correlation between crystal structure and electro-optical properties. Potential energy calculations have provided some insights into the different crystal structure types formed within this family of materials, particularly in relation to the competing influences of electrostatic and steric factors.

While the structural information determined from powder diffraction is not, in general, as accurate as that which could be determined from single-crystal diffraction on the same material, we nevertheless emphasize that for understanding details of the intermolecular packing arrangements and characterization of different structure types, the structural information determined from powder diffraction data is perfectly adequate. Furthermore, it is important to emphasize the following inherent advantages of structural analysis by powder diffraction data, some of which may find particular relevance in establishing (as in the present paper) relationships between the structure and properties (and ultimately potential applications) of crystalline materials: (i) many crystalline materials (as in the case of 3 ) cannot be prepared as single crystals of sufficient size and quality for single-crystal X-ray diffraction studies; (ii) in structure determination from powder diffraction data, the structure obtained is inherently representative of the bulk material; and (iii) comparison of the diffraction data of a thin film of the material of interest and the powder diffraction pattern of the corresponding bulk material may provide opportunities to obtain a structural model for the thin film (this aspect is particularly relevant in the context of those materials applications in which the material of interest is deposited as a thin film).

\section{ACKNOWLEDGMENTS}

We are grateful to Wyeth-Ayerst (postdoctoral fellowship to E.T.), EPSRC (general support to K.D.M.H.), and MURST (research grant to G.B.).

\section{REFERENCES}

1. N. C. Greenham, S. C. Moratti, D. D. C. Bradley, R. H. Friend, and A. B. Holmes, Nature 365, 628 (1993).

2. J. H. Burroughes, D. D. C. Bradley, A. R. Brown, R. N. Marks, K. Mackay, R. H. Friend, P. L. Burn, and A. B. Holmes, Nature 347, 539 (1990).

3. C. W. Tang and S. A. Van Slyke, Appl. Phys. Lett. 51, 913 (1987).

4. J. R. Sheats, H. Antoniadis, M. Hueschen, W. Leonard, J. Miller, R. Moon, D. Roitman, and A. Stocking, Science 273, 884 (1996).

5. A. Kraft, A. G. Grimsdale, and A. B. Holmes, Angew. Chem., Int. Ed. 37, 402 (1998).

6. G. Leising, S. Tasch, and W. Graupner, in "Handbook of Conductive Polymers" (T. A. Skotheim, R. L. Elsenbaumer, and J. R. Reynolds, Eds.), p. 847. Dekker, New York, 1998.

7. M. Berggren, O. Inganäs, G. Gustafsson, J. Rasmusson, M. R. Andersson, T. Hjertberg, and O. Wennerström, Nature 372, 444 (1994).

8. J. D. Anderson, E. M. McDonald, P. A. Lee, M. L. Anderson, E. L. Ritchie, H. K. Hall, T. Hopkins, E. A. Mash, J. Wang, A. Padias, S. Thayumanavan, S. Barlow, S. R. Marder, G. E. Jabbour, S. Shaheen, B. Kippelen, N. Peyghambarian, R. M. Wightman, and N. R. Armstrong, J. Am. Chem. Soc. 120, 9646 (1998).

9. J. Stampfl, S. Tasch, G. Leising, and U. Scherf, Synth. Met. 71, 2125 (1995).

10. F. Garnier, Acc. Chem. Res. 32, 209 (1999).

11. P. F. Van Hutten, V. V. Krasnikov, and G. Hadziioannou, Acc. Chem. Res. 32, 257 (1999).

12. J. L. Brédas, J. Cornil, D. Beljonne, D. A. Dos Santos, and Z. Shuai, Acc. Chem. Res. 32, 267 (1999). 
13. L. Antolini, E. Tedesco, G. Barbarella, L. Favaretto, G. Sotgiu, M. Zambianchi, D. Casarini, G. Gigli, and R. Cingolani, J. Am. Chem. Soc. 122, 9006 (2000).

14. L. G. Vorontsova, Zh. Strukt. Khim. 7, 240 (1966).

15. E. Lukevics, P. Arsenyan, S. Belyakov, J. Popelis, and O. Pudova, Organometallics 18, 3187 (1999).

16. G. Barbarella, O. Pudova, C. Arbizzani, M. Mastragostino, and A. Bongini, J. Org. Chem. 63, 1742 (1998).

17. G. Gigli, G. Barbarella, L. Favaretto, F. Cacialli, and R. Cingolani, Appl. Phys. Lett. 75, 439 (1999).

18. B. M. Kariuki, H. Serrano-González, R. L. Johnston, and K. D. M. Harris, Chem. Phys. Lett. 280, 189 (1997).

19. K. D. M. Harris, R. L. Johnston, and B. M. Kariuki, GAPSS (Genetic Algorithm for Powder Structure Solution), University of Birmingham, 1997.

20. K. D. M. Harris, R. L. Johnston, and B. M. Kariuki, Acta Crystallogr. A 54, 632 (1998).

21. A. C. Larson and R. B. Von Dreele, Los Alamos Lab. Report No. LA-UR-86-748, 1987.

22. Cerius ${ }^{2}$, Version 4.2 Materials Science, Molecular Simulations Inc., San Diego, CA, 2000.

23. A. T. Hagler, E. Huler, and S. Lifson, J. Am. Chem. Soc. 96, 5319 (1974).

24. A. T. Hagler and S. Lifson, J. Am. Chem. Soc. 96, 5327 (1974).

25. S. Lifson, A. T. Hagler, and P. Dauber, J. Am. Chem. Soc. 101, 5111 (1979)

26. A. T. Hagler, S. Lifson, and P. Dauber, J. Am. Chem. Soc. 101, 5122 (1979).

27. A. T. Hagler, P. Dauber, and S. Lifson, J. Am. Chem. Soc. 101, 5131 (1979).

28. D. H. Kitson and A. T. Hagler, Biochemistry 27, 5246 (1998).
29. D. H. Kitson and A. T. Hagler, Biochemistry 27, 7176 (1998).

30. P. Dauber-Osguthorpe, V. A. Roberts, D. J. Osguthorpe, J. Wolff, M. Genest, and A. T. Hagler, Proteins: Struct. Funct. and Genet. 4, 31 (1998).

31. N. Karasawa and W. A. Goddard III, J. Phys. Chem. 93, 7320 (1989).

32. C. Ziegler, in "Handbook of Organic Conductive Molecules and Polymers" (H. S. Nalwa, Ed.), Vol. 3, p. 677. Wiley, New York, 1997.

33. T. Granlund, M. Theander, M. Berggren, M. Andersson, A. Ruzeckas, V. Sundström, G. Björk, M. Granström, and O. Inganäs, Chem. Phys. Lett. 288, 879 (1998).

34. P. Barta, F. Cacialli, R. H. Friend, and M. Zagórska, J. Appl. Phys. 84, 6279 (1998).

35. G. Barbarella, et al., unpublished results.

36. M. Anni, G. Gigli, V. Paladini, R. Cingolani, G. Barbarella, L. Favaretto, G. Sotgiu, and M. Zambianchi, Appl. Phys. Lett. 77, 2458 (2000).

37. G. Barbarella, L. Favaretto, G. Sotgiu, M. Zambianchi, A. Bongini, C. Arbizzani, M. Mastragostino, M. Anni, G. Gigli, and R. Cingolani, J. Am. Chem. Soc. 122, 11971 (2000).

38. K. D. M. Harris and M. Tremayne, Chem. Mater. 8, 2554 (1996).

39. D. M. Poojary, and A. Clearfield, Acc. Chem. Res. 30, 414 (1997).

40. A. Meden, Croat. Chem. Acta 71, 615 (1998).

41. K. D. M. Harris, M. Tremayne, and B. M. Kariuki, Angew. Chem., Int. Ed. 40, 1626 (2001).

42. P.-E. Werner, L. Eriksson, and M. Westdahl, J. Appl. Crystallogr. 18, 367 (1985).

43. B. M. Kariuki, P. Calcagno, K. D. M. Harris, D. Philp, and R. L. Johnston, Angew. Chem. Int. Ed. 38, 831 (1999).

44. E. Tedesco, G. W. Turner, K. D. M. Harris, R. L. Johnston, and B. M. Kariuki, Angew. Chem., Int. Ed. 39, 4488 (2000). 